\title{
Seroprevalencia de virus hepatitis B en niños con cáncer en tratamiento quimioterápico en 6 hospitales de Santiago de Chile
}

\author{
Marcela Zubieta ${ }^{1,8}$, María E Santolaya 2,8, Carmen Hurtado ${ }^{3 a}$, \\ Ana M Alvarez ${ }^{4,8}$, Carmen L Avilés,8, Ana Becker6,8, \\ Javier Brahm ${ }^{3}$, Carmen Salgado ${ }^{1,8}$, Pamela Silva ${ }^{5,8}$, \\ Santiago Topelberg, 7,8 , Juan Tordecilla,8, Mónica Varas ${ }^{4,8}$, \\ Milena Villarroel ${ }^{2,8}$,Tamara Viviani 6,8 . \\ Seroprevalence of hepatitis $B$ virus \\ in children with cancer under \\ chemotherapy in 6 hospitals \\ of Santiago, Chile
}

Background: Children under oncological therapy are at risk of infection by hepatitis B virus (HBV). Aim: To determine the prevalence of infection of HBV in children with cancer who have undergone chemotherapy or have had an hematopoietic stem cell transplant. Material and methods: Collaborative, multi-centric study. Serum samples were collected from 281 children with cancer and episodes of febrile neutropenia, from 6 hospitals belonging to the public health network in the Metropolitan Region, between June 2004 and August 2006. These samples were stored at $-70^{\circ} \mathrm{C}$. In September 2006, 200 samples were randomly chosen and 170 analyzed to determine hepatitis B virus surface antigen (HBsAg) and anticore total antibodies (anti HBc) by fluorescent EUSA (Enzyme Linked Immunosorbent Assay). In five cases in whom a low volume of sample was available, only one marker was studied (HBsAg in two and anti HBc in three). Results: Samples came from children aged 4 months to 18 years, 104 males (61\%). They had received an average of 38 transfusions (range 3-107) from 65 donors (range 3-345). Twelve children were found positive for some marker of HBV: HBsAg in three (1.8\%) and anti HBc in ten (7\%). In 5 patients that had negative HBsAg and positive anti HBc, anti surface antigen antibodies (anti HBs) were determined and resulted positive in four. Conclusions: The prevalence of $\mathrm{HBV}$ in this sample was $7 \%$ if both serologic markers are considered and $1.8 \%$ if only HBsAg is considered (Rev Méd Chile 2009; 137: 906-11).

(Key words: Antineoplastic protocols; Blood component transfusion; Hepatitis B virus)

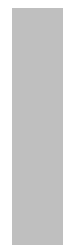

\footnotetext{
Recibido el 24 de noviembre, 2008. Aceptado el 26 de mayo, 2009.

Trabajo financiado por Fundación Nuestros Hijos. Proyecto Fondecyt \#1040907.

${ }^{1}$ Hospital Exequiel González Cortés; ${ }^{2}$ Hospital Luis Calvo Mackennna; ${ }^{3}$ Laboratorio de Gastroenterología, Hospital Clínico Universidad de Chile; ${ }^{4}$ Hospital San Juan de Dios; ${ }^{5}$ Hospital San Borja Ariarán; ${ }^{6}$ Hospital Sótero del Río; ${ }^{7}$ Hospital Roberto del Río; ${ }^{8}$ Subcomité de Enfermedades Infecciosas, Programa Infantil Nacional de Drogas Antineoplásicas, Santiago, Chile. aQuímica Farmacéutica
}

Correspondencia a: Dra. Marcela Zubieta A. La Fragua 2053, Lo Barnechea. Teléfono: (562)4805960. Fax: (562)5568840. E mail: mzubieta@fnh.cl 
L os niños que reciben terapia oncológica están expuestos a infectarse o reactivar una infección por virus de hepatitis $B(\mathrm{VHB})^{1}$. Estos niños reciben transfusiones de productos sanguíneos en forma frecuente ${ }^{2}$ y además están expuestos al riesgo de adquirir esta infección mediante transmisión horizontal ${ }^{3}$.

La transmisión de infecciones virales a través de transfusiones sanguíneas es actualmente prevenida seleccionando donantes sin factores de riesgo y estudiando sistemáticamente la sangre donada, de manera que las personas infectadas son eliminadas como potenciales donantes y las muestras infectadas son desechadas. La transmisión horizontal se previene cumpliendo estrictamente las normas de control de infección intrahospitalaria.

A pesar de estas medidas, existe un riesgo residual de transmitir esta infección a través de transfusiones de productos sanguíneos, el cual se asocia principalmente al periodo de incubación de la enfermedad, periodo en el cual los marcadores serológicos de infección podrían no ser detectados y a donantes de sangre que presentan una infección oculta por VHB caracterizada por ausencia de marcadores serológicos asociadas por lo general con una baja carga de $\mathrm{ADN}^{4-6}$.

Chile es considerado un país de prevalencia baja de infección por VHB, según diferentes estudios ésta fluctúa entre 0\% y 0,3\%. Desde 1982 se realiza en nuestro país la pesquisa obligatoria de antígeno de superficie (HBsAg) en todos los bancos de sangre; la seroprevalencia de portación en donantes de sangre en nuestro medio ha sido reportada en promedio en $0,2 \%$ entre los años 1999 y 2004, sin embargo, en donantes seleccionados sin factores de riesgo ésta es de 0,035\% (Zapata R. 2005, estudio no publicado). La prevalencia de anticuerpos anticore total (Anti HBc) en un estudio efectuado en 500 donantes el año 1980 fue de $5 \%{ }^{7-9}$.

La infección por VHB en individuos con cáncer se caracteriza por una alta tasa de cronicidad y se relaciona con mayor riesgo de daño hepático, de desarrollar un cáncer secundario, mayor posibilidad de recaída de su enfermedad oncológica, un riesgo epidemiológico especial para sus familiares y los trabajadores de la salud que atienden a estos pacientes y además deterioro en la calidad de vida de los sobrevivientes ${ }^{3,7}$.
En los últimos años se ha diagnosticado hepatitis B en forma esporádica en niños enfermos de cáncer, con excepción del año 2006, en que se informó un brote de VHB en un centro oncológico privado de la ciudad de Santiago ${ }^{10}$.

No existen publicaciones de seroprevalencia de infección por VHB en niños en tratamiento por cáncer en nuestro país, por lo que nuestro objetivo fue determinar la prevalencia de infección por VHB en estos niños sometidos a tratamiento quimioterápico o trasplante de precursores hematopoyéticos (TPH).

\section{MATERIAL Y MÉTODO}

Entre junio 2004 y agosto 2006 se realizó un estudio prospectivo, colaborativo y multicéntrico en el que participaron 6 hospitales de la Región Metropolitana: Dr. Exequiel González Cortés, Dr. Luis Calvo Mackennna, San Juan de Dios, San Borja Arriarán, Dr. Sótero del Río y Dr. Roberto del Río. Durante este periodo se recolectaron muestras de sangre de 281 niños menores de 18 años provenientes de estos hospitales. Las muestras fueron congeladas a $-70^{\circ} \mathrm{C}$ para posterior análisis.

En el momento de la extracción de la muestra de sangre, todos los niños se encontraban en tratamiento quimioterápico o habían recibido un TPH y estaban cursando con un episodio de neutropenia febril.

En septiembre de 2006, luego de obtener la aprobación del Comité de Ética del Ministerio de Salud (MINSAL), 200 de estas muestras fueron tomadas al azar para determinar prevalencia de infección por VHB, a través de la determinación de HBsAg y Anti HBc.

El HBsAg y Anti $\mathrm{HBC}$ se procesaron por técnica de enzimoinmuno ensayo de fluorescencia (micropartículas IMx Lab. Abbott). En los pacientes con resultados de HBsAg negativo y anti HBc positivo, se determinó anticuerpos anti HBsAg (Anti HBs) por igual metodología.

Todas las muestras que fueron positivas se analizaron en duplicado y se solicitaron muestras actuales de los niños sobrevivientes para verificar los primeros resultados. Todos los exámenes de marcadores virales se realizaron en el Laboratorio de Gastroenterología del Hospital Clínico de la Universidad de Chile. 
Estadística. Se utilizó test de t-student y $\mathrm{Chi}^{2}$ para el análisis de los resultados. Se consideró significativo un valor de $\mathrm{P}<0,05$.

\section{Resultados}

De las 200 muestras tomadas al azar, 30 fueron excluidas: 15 eran muestras repetidas del mismo paciente, 6 por falta de información, 5 con cantidad de muestra insuficiente y 4 por ser pacientes con TPH de causa no oncológica.

Se estudiaron por lo tanto muestras de 170 niños. La distribución de la muestra fue representativa de la población de niños en tratamiento en cada uno de los 6 centros en términos porcentuales ( $p=0,69$ ). Ciento cuatro de los 170 niños estudiados comespondieron al género masculino (61\%), con una edad promedio de 6 años (rango 4 meses - 18 años).

En 165 de las 170 muestras se determinó HBsAg y Anti HBc, en 5 pacientes sólo se estudió un marcador dado que la cantidad de la muestra fue insuficiente para estudiar ambos, en 2 de éstos sólo se determinó HBsAg y en 3 sólo, Anti HBc. En los 5 pacientes en que se estudió sólo 1 marcador, éste resultó negativo.

Del total de niños estudiados, en 12 se encontró positivo algún marcador de VHB (7\%): en 2 sólo HBsAg, en 9 sólo Ac HBc y 1 resultó positivo a ambos marcadores. El HBsAg fue positivo en 3/167 niños (1,8\%), y Anti HBc resultó positivo en 10/168 muestras (5,9\%) (Tabla 1$)$.
En 5 de los 9 niños con Anti HBc positivo y HBsAg negativo en el estudio inicial, se pudo determinar anticuerpo anti HBs, siendo positivo en 4 de éstos. En los 4 restantes no se pudo determinar este marcador dado que la cantidad de la muestra fue insuficiente (Tabla 2).

Características de los 12 pacientes VHB positivos. Siete mujeres; promedio de edad 4 años (rango de 5 meses a 14 años); enfermedad de base: 6 leucemia linfoblástica aguda (2 de ellos sometidos a TPH), 3 leucemia mieloide aguda, 2 rabdomiosarcoma y 1 linfoma no Hodgkin B (Tabla 2); provenientes de 4 de los 6 hospitales involucrados en el estudio (Tabla 1). Ninguno había recibido vacuna VHB del Programa Nacional de Inmunizaciones. Los pacientes habían recibido en promedio 38 transfusiones (rango 3 a 107), de un promedio de 65 donantes (rango 3- 345).

Evolución de marcadores en el tiempo. A 8 de los 9 niños que presentaron Anti $\mathrm{HBc}$ positivo como único marcador en el estudio inicial, se les repitió este marcador en una nueva muestra un año después de la primera determinación. Sólo en 2 casos este marcador se encontró positivo (Tabla 2).

No se repitieron los marcadores a los 3 niños que fueron positivos para HBsAg, ya que ellos fallecieron como consecuencia de su enfermedad de base. En ninguno de ellos existía el reporte de una hepatitis viral asociada, según análisis retrospectivo de la ficha clínica.

Tabla 1. D istribución por hospital de niños con H BsAg o antiH Bc positivo

\begin{tabular}{|lccccc|}
\hline H ospital & no & H BsAg (+) & AntiH Bc (+) & Total (+) & (\%) \\
\hline 1 & 21 & 0 & 0 & 0 & $(0)$ \\
2 & 69 & 0 & 5 & 5 & $(7,2)$ \\
3 & 37 & 3 & 2 & 4 & $(8,1)$ \\
4 & 11 & 0 & 1 & 1 & $(9,1)$ \\
5 & 17 & 0 & 0 & 0 & $(0)$ \\
6 & 15 & 0 & 2 & 2 & $(13,3)$ \\
Total & 170 & 3 & 10 & 12 & $(7)$ \\
\hline
\end{tabular}


Tabla 2. Características clínicas y epidemiológicas de los pacientes con algún marcador positivo para el VH B, al diagnóstico y durante el seguimiento

\begin{tabular}{|c|c|c|c|c|c|c|c|c|c|c|}
\hline \multirow[b]{2}{*}{ Gén. } & \multirow[b]{2}{*}{$\begin{array}{l}E . \\
\text { (m) }\end{array}$} & \multirow[b]{2}{*}{ Tipo de cáncer } & \multicolumn{3}{|c|}{ D iagnóstico } & \multicolumn{5}{|c|}{ Seguimiento } \\
\hline & & & $\begin{array}{l}\text { Anti } \\
\text { H Bc }\end{array}$ & $\begin{array}{c}\text { H Bs } \\
\text { Ag }\end{array}$ & $\begin{array}{l}\text { Anti } \\
\text { H Bs }\end{array}$ & $\begin{array}{l}\text { I.T. } \\
\text { (m) }\end{array}$ & $\begin{array}{l}\text { Anti } \\
\text { H BC }\end{array}$ & $\begin{array}{c}\text { H Bs } \\
\text { Ag }\end{array}$ & $\begin{array}{l}\text { Anti } \\
\text { H Bs }\end{array}$ & Est. \\
\hline M & 50 & LNHB & + & - & NR & & - & - & + débil & $\mathrm{V}$ \\
\hline M & 120 & ШA & + & - & - & 14 & - & - & - & V \\
\hline $\mathrm{F}$ & 21 & LMA & + & - & NR & 10 & + & - & - & V \\
\hline $\mathrm{F}$ & 35 & LA recaída & + & - & NR & 5 & + & - & - & $\mathrm{V}$ \\
\hline M & 28 & LA RM & + & - & + & 9 & - & - & - & $\mathrm{V}$ \\
\hline M & 96 & LMA & + & - & + & 11 & - & - & - & $\mathrm{V}$ \\
\hline M & 168 & LAA recaída & + & - & + & 24 & - & - & - & $\mathrm{V}$ \\
\hline $\mathrm{F}$ & 5 & LAA congénita & + & - & + & & NR & NR & NR & Fall \\
\hline $\mathrm{F}$ & 12 & Rabd IV & + & - & NR & 32 & - & - & + débil & $\mathrm{V}$ \\
\hline F & 81 & LA & + & + & NR & & NR & NR & NR & Fall \\
\hline F & 96 & LMA & - & + & NR & & NR & NR & NR & Fall \\
\hline $\mathrm{F}$ & 70 & Rabd. IV & - & + & NR & & NR & NR & NR & Fall \\
\hline
\end{tabular}

Anti HBc: Anticuerpos anti Core total hepatitis B

Gén: género

Anti HBs: Anticuerpos anti antígeno de superficie hepatitis B

IT: intervalo de tiempo

M: masculino

F: femenino

NR: no realizado

LNHB: linfoma no Hodgkin B

\section{DisCUSIÓN}

Los estudios de seroprevalencia de VHB en niños inmunocomprometidos sometidos a múltiples transfusiones de productos sanguíneos son de difícil interpretación. El hallazgo de anticuerpos positivos (Anti HBc, anti HBs) en ausencia de HBsAg en esta población, podría deberse a una infección pasada por VHB con resolución de la infección o ser consecuencia de las transfusiones de sangre de donantes que alguna vez estuvieron infectados por el VHB (traspaso de anticuerpos). En los bancos de sangre en nuestro país, se determina sólo HBsAg como método de tamizaje.

Un estudio efectuado en Chile en el año 1980 demostró $5 \%$ de positividad para Anti $\mathrm{HBc}$ en donantes de sangre, porcentaje similar al encontrado en esta población (5,9\%) ${ }^{7}$. En nuestro estudio, el Anti HBc permaneció positivo luego de 10-15 meses de seguimiento en sólo 2 de los 8 pacientes que presentaron Anti $\mathrm{HBc}$ positivo como único
HBsAg: Antígeno de superficie hepatitis B

E: edad

Est: estado al finalizar estudio

$\mathrm{V}$ : vivo

Fall: fallecido

LA: leucemia linfoblástica aguda

LMA: leucemia mieloblástica aguda

Rabd. IV: rabdomiosarcoma etapa IV marcador en el estudio inicial (Tabla 2). Aunque estos pacientes pudieran haber cursado una infección por VHB previo a su enfermedad oncológica y presentar desaparición de anticuerpos verdaderos durante el curso de la enfermedad debido a inmunosupresión, la tasa de infección de $0 \%$ encontrada en población chilena menor de 15 años $^{11}$, hace poco probable esta posibilidad. También podría pensarse que estos niños se contagiaron durante el tratamiento, y resolvieron la infección, sin embargo la inmunosupresión como consecuencia de la enfermedad de base en el caso de las leucemias o al tratamiento quimioterápico intensivo en cualquier tipo de cáncer y la evolución natural de la enfermedad en los niños, hace muy poco probable esta situación. En una serie de 119 niños enfermos de cáncer en tratamiento en Polonia antes del año 1992, en que la prevalencia de VHB en un seguimiento de 6 meses fue de $62,2 \%$, sólo en 4 de $74(5,4 \%)$ casos infectados por VHB ocurrió eliminación espontánea del virus ${ }^{3}$. Dado 
estos antecedentes pensamos que lo más probable es que la seroprevalencia de Anti HBc encontrado en este estudio sea consecuencia de anticuerpos recibidos de los donantes de sangre.

Una limitación de este trabajo es que no se contó con la posibilidad de efectuar estudios moleculares, determinación de reacción en cadena de polimerasa (PCR) o carga viral para VHB, lo que nos hubiera permitido descartar infección oculta en pacientes Anti HBc positivos y HBs Ag negativo. Tampoco tuvimos la posibilidad de conocer la serología de las madres de los pacientes lo que habría ayudado a dilucidar si el contagio fue previo a las transfusiones recibidas.

En nuestro estudio 3 niños tenían $\mathrm{HBsAg}$ repetidamente positivo y 2 de ellos no tenían Anti HBc. Es probable que los 2 niños no hayan podido crear anticuerpos o se encontraban en periodo de incubación de la infección por VHB.

Estos 3 niños con HBsAg positivo estaban en tratamiento en el mismo centro y recibieron quimioterapia en forma contemporánea en algún periodo de su enfermedad. Aunque no puede descartarse con seguridad una transmisión horizontal entre ellos, nos parece poco probable, dado que en los hospitales en Chile se cumplen las normas en relación a precauciones estándar frente a exposición a sangre y fluidos corporales ${ }^{12}$, en consecuencia la posibilidad de contagio más probable parece ser vía transfusión sanguínea.

Sin considerar los pacientes que pudieran haber estado cursando con una infección oculta, cuyo único marcador en este estudio fue Anti $\mathrm{HBc}$, la seroprevalencia de infección de 1,8\%, encontrada en nuestro estudio es claramente superior al promedio reportado en la población general de Chile ${ }^{7-9}$. En esta subpoblación de niños con cáncer y en tratamiento quimioterápico que no recibieron vacuna contra VHB previo a su enfermedad, existe un mayor riesgo de infección por este virus y un mayor riesgo de evolucionar al estado de portador. La infección por VHB durante el tratamiento quimioterápico puede conllevar que el virus permanezca en estado latente 0 de inmunotolerancia; sin embargo, durante la quimioterapia de mantención y en los periodos posteriores a ésta, al recuperar su capacidad de respuesta inmunológica, el virus puede reactivarse y provocar injuria hepatocelular. El riesgo de reactivación después de quimioterapia varía según las diferentes series, reportándose en rango de $14 \%$ a $72 \%{ }^{1}$. Estos antecedentes, seroprevalencia claramente más alta con respecto a la población general de Chile y mayor riesgo de morbilidad, nos obligan a reforzar las medidas preventivas en esta población de niños inmunocomprometidos, que consisten en insistir en la vacunación sistemática de ellos y en mejorar el tamizaje de VHB en donantes de sangre.

En relación a la vacunación contra el VHB, el año 2005 se incorporó en Chile esta vacuna en el Programa Nacional de Inmunizaciones (PNI) a la edad de 2, 4 y 6 meses. Sin embargo, no todos los niños actualmente en tratamiento por una patología oncológica, alcanzaron por su edad a beneficiarse con este programa. Posteriormente, en octubre de 2006, el MINSAL inició un Programa especial de vacunación para niños con inmunodeficiencias adquiridas, que es parte del PNI, y que incluye, entre otras vacunas, la del $\mathrm{VHB}^{13}$. La vacuna contra VHB ha mostrado ser eficaz en niños sometidos a quimioterapia ${ }^{3,14}$.

El riesgo de hepatitis postransfusional se puede disminuir con la determinación conjunta de HBsAg y Anti HBc en donantes con infección clásica o residual, esta última definida como aquellos con HBsAg negativo y Anti HBc positivo; algunos países latinoamericanos ya han adoptado esta práctica. Con el objetivo de detectar individuos cursando el denominado "periodo ventana" o en la etapa crónica y oculta de la infección en que ambos marcadores pueden resultar negativos y ser fuentes potenciales de transmisión de la infección, algunos países desarrollados evalúan las donaciones sanguíneas mediante el uso de técnicas de amplificación de ácidos nucleicos que han demostrado alta sensibilidad $^{15}$.

En pacientes con antecedentes de hepatitis crónica o historia de infección por $\mathrm{VHB}$, se recomienda iniciar tratamiento profiláctico con antivirales análogos de nucleósido, para prevenir reactivación por quimioterapia ${ }^{16,17}$.

Nuestros resultados, muestran que la seroprevalencia de VHB en niños en tratamiento por cáncer es de $7 \%$ si se consideran ambos marcadores (HBsAg y Anti $\mathrm{HBc}$ ), y de 1,8\%, si se considera sólo el HBsAg, superior al reportado en estudios en población general de Chile, lo que claramente obliga a tomar medidas preventivas y terapéuticas en esta población. 
Agradecimientos

Agradecemos la colaboración de la. Sra. Jazmine Fernández (E.U. Fundación Nuestros Hijos) y de la Srta. Rosario Berríos (Secretaria Unidad Investigación Hospi-

\section{REFERENCIAS}

1. Mindikoglu A, Regev A, Schiff E. Hepatitis B Virus Reactivation After Cytotoxic Chemotherapy: The disease and its prevention. Review. Clin Gastroenterol Hepatol 2006; 4: 1076-81.

2. Kebudi R, Ayan I, Yilmaz G, Akici F, Gorguin O, Badur S. Seroprevalence of hepatitis $B$, hepatitis $C$, and Human Inmunodeficiency Virus Infections in Children with cancer at diagnosis and following therapy in Turkey. Med Ped Oncol 2000; 34: 102-5.

3. Styczynski J, Wysocki M, Koltan S, Kurylak A. Epidemiologic aspects and preventive strategy of hepatitis B and C viral infections in children with cancer. Pediatr Infect Dis J 2001; 20: 1042-9.

4. Dodd R, Notari I, Stramer S. Current prevalence and incidence of infectious disease markers and estimated window-period risk in the American Red Cross blood donor population. Transfusion 2002; 42: 975-9.

5. Pillonel J, Laperche S, Saura C, Desenclos J-C, Courouce A-M, and the Transfusion-Transmissible Agents Working Group of the French Society of Blood Transfusion. Trend in residual risk of transfusion-transmitted viral infections in France between 1992-2000. Transfusion 2002; 42: 980-8.

6. Del Rey-Pineda G. Aplicación de nuevas técnicas de Biología Molecular a la virología. Detección de tamizaje en bancos de sangre. Gac Med Mex 2004; 140, Suplemento $\mathrm{N}^{0} 3$ : 73-5.

7. Zunino E. Epidemiología de la hepatitis B en Chile y esquemas de vacunación en Latinoamérica. Rev Chil Infectol 2002; 19: 140-55.

8. Pereira A, Valenzuela M, Mora J, Vera L Situación actual de la hepatitis B en Chile. Rev Méd Chile 2008; 136: 725-32.

9. Ministerio de Salud. Encuesta de Salud Chile 2003. Disponible en: http://epi.minsal.cl/epi/html/invest/ ENS/ENS.htm. tal Luis Calvo Mackenna) en esta investigación y el análisis estadístico efectuado por el Sr. Miguel Ángel Cumsille, Estadístico de la Facultad de Medicina de la Universidad de Chile.

10. Torres J. Hepatitis B Chile. Disponible en: http:// www.promedmail.org/ ISID. Archive Number: 20060818.2308. 18 agosto 2006 (Consultado el 31 de octubre de 2008).

11. Silveira T, Fonseca J, Rivera L, Fay O, Tapia R, Santos J ET AL. Hepatitis B seroprevalence in Latin America. Rev Panam Salud Pública 1999; 6: 378-83.

12. Siegel JD, Rhinehart E, Jackson M, Chiarelo L and the Healthcare Infection Control Practices Advisory ComMTTEE, 2007 Guideline for Isolation Precautions: Preventing Transmission of Infectious Agents in Healthcare Settings, June 2007. Disponible en: http:/ / www.cdc.gov/ncidod/dhqp/pdf/isolation2007.pdf/ (Consultado el 31 de octubre de 2008).

13. Ministerio de Salud de Chile. División de Prevención y Control de Enfermedades. Departamento de Enfermedades Transmisibles. Programa Nacional de Inmunizaciones. Vacunacion de niños con inmunosupresión adquirida excluido VIH/SIDA. Ord. B211. № 3272. 5 de octubre de 2006.

14. Yetgin S, Tavil B, Aytac S, Kuskonmaz B, Karna G. Unexpected protection from infection by two booster hepatitis B virus vaccination in children with acute lymphoblastic leukemia. Leuk Res 2007; 31: 496-7.

15. Gutiérrez C, León G, Liprandi F, Pujol F. Bajo impacto de la infección silente por el virus de la hepatitis B en la incidencia de hepatitis postransfusional en Venezuela. Rev Panam Salud Pública 2001; 10: 382-7.

16. Mindikoglu AL, Regev A, Schiff ER. Hepatitis B Virus After Cytotoxic Chemotherapy: The disease and its prevention. Review. Clin Gastroenterol Hepatol 2006; 4: 1076-81.

17. El-Sayed MH, Shanab G, Karim AM, Ez-Tawil A, Biack A, Dixon JS. Lamivudine facilitates optimal chemotherapy in hepatitis B virus-infected children with hematology malignancies. Ped Hemat Oncol 2004; 21: 145-56. 